\title{
QPSO-based Energy-aware Clustering Scheme in the Capillary Networks for Internet of Things Systems
}

\author{
Liumeng Song*, Kok Keong Chai*, Yue Chen*, Jonathan Loo ${ }^{\dagger}$, Shihab Jimaa ${ }^{\ddagger}$, John Schormans* \\ * School of Electronic Engineering and Computer Science, Queen Mary University of London, London, UK \\ $\dagger$ School of Engineering and Information Sciences, Middlesex University, London, UK \\ ‡ Electrical and Computer Engineering Department, Khalifa University of Science, Technology \& Research, Sharjah, UAE \\ Email: \{1.song, michael.chai, yue.chen\}@qmul.ac.uk, j.loo@mdx.ac.uk, saj@kustar.ac.ae, john.schormans@qmul.ac.uk
}

\begin{abstract}
Energy efficiency is a crucial challenge in clusterbased capillary networks for Internet of Things (IoT) systems, where the cluster heads (CHs) selection has great impact on the network performance. It is an optimization problem to find the optimum number of $\mathrm{CHs}$ as well as which devices are selected as CHs. In this paper, we formulate the clustering problem into the CHs selection procedure with the aim of maximizing the average network lifetime in every round. In particular, we propose a novel CHs selection scheme based on QPSO and investigate how effective it is to prolong network lifetime and reserve the overall battery capacity. The simulation results prove that the proposed QPSO outperforms other evolutionary algorithms and can improve the network lifetime by almost $10 \%$.

Index Terms-IoT systems, cluster, QPSO, energy efficiency, network lifetime, battery capacity.
\end{abstract}

\section{INTRODUCTION}

The Internet of Things (IoT) system is viewed to have potential to improve the operational efficiency of many industrial applications [1]. The capillary networks were introduced to improve reliable and energy efficient communications for the IoT systems. The capillary networks are specific local networks which consist of a group of wireless devices to be connected to other communication infrastructure such as mobile networks [2]. Although capillary networks can provide reliable connectivity to devices within a specific local area, energy efficiency of the long-haul transmission is a main challenging issue.

The clustering protocol was then proposed as an energy efficient cross-layering technique to solve the aforementioned issue, which organizes devices into several clusters to reduce both long-haul distance and the data volume [3]. In particular, a device is selected as cluster head $(\mathrm{CH})$ in each cluster, which consequently collects data from all cluster members (CMs), eliminates the correlated data and transmits the aggregated data to the sink via communication infrastructure networks.

From an optimization perspective, clustering is a kind of NP-hard grouping problem [4]. Particularly, evolutionary algorithms are metaheuristics widely believed to be effective on NP-hard problems, being able to provide near-optimal solutions to such problems in a reasonable time [5]. Evolutionary algorithms such as [6] [7] [8] have been applied in centralized clustering recently. In [6], the authors proposed an energyaware clustering for wireless sensor networks using particle swarm optimization (PSO) algorithm which is implemented at the base station, with the objective of simultaneously minimizing the intra-cluster distance and optimizing the energy consumption of the networks. The simulation results suggest that the performance of PSO-based algorithm outperforms LEACH (Low-Energy Adaptive Clustering Hierarchy) [9]. In [7], the authors presented a dynamic clustering method with multi-objectives that automatically determines the optimum number of clusters in a network using binary particle swarm optimization (BPSO). Simulation results demonstrate that the proposed protocol can achieve an optimal number of clusters, as well as prolong the network lifetime and increase the data delivery at the base station. In [8], the authors proposed a quantum genetic algorithm (QGA) based clustering protocol to determine the $\mathrm{CH}$ selection, aiming at the energy consumption balance between CMs and CHs.

Quantum-inspired particle swarm optimization (QPSO) combines the advantages of the quantum computing theory and the evolutionary algorithm. Compared with PSO, QPSO adopts novel rotation angle and quantum bit techniques so it has the characteristics of strong searching capability, rapid convergence, short-computing time, and small-population size [10]. In addition, the network lifetime is significantly affected by the characteristics of selected $\mathrm{CHs}$, e.g. the residual energy, the long-haul distance and the total number of selected CHs [11], which indicates that how to apply the evolutionary algorithms to converge an optimal solution of the $\mathrm{CHs}$ selection problem becomes a crucial challenge. This consideration motivated our research work to investigate the ability of QPSO.

In this paper, the clustering problem is firstly formulated into the $\mathrm{CHs}$ selection procedure with the aim of maximizing average network lifetime. Then we apply QPSO in CHs selection and investigate how effective it is in prolonging network lifetime as well as reserving the overall battery capacity among the whole networks.

The rest of this paper is organized as follow. Section II introduces the system model, the energy model and the problem formulation. In Section III, we explain the QPSO algorithm 
in detail and how to apply QPSO to obtain the optimal CHs coalition with the objective of network lifetime longevity. Simulation results are provided in Section IV, and conclusions are drawn in Section V.

\section{System Model AND PROBlem Formulation}

\section{A. System model}

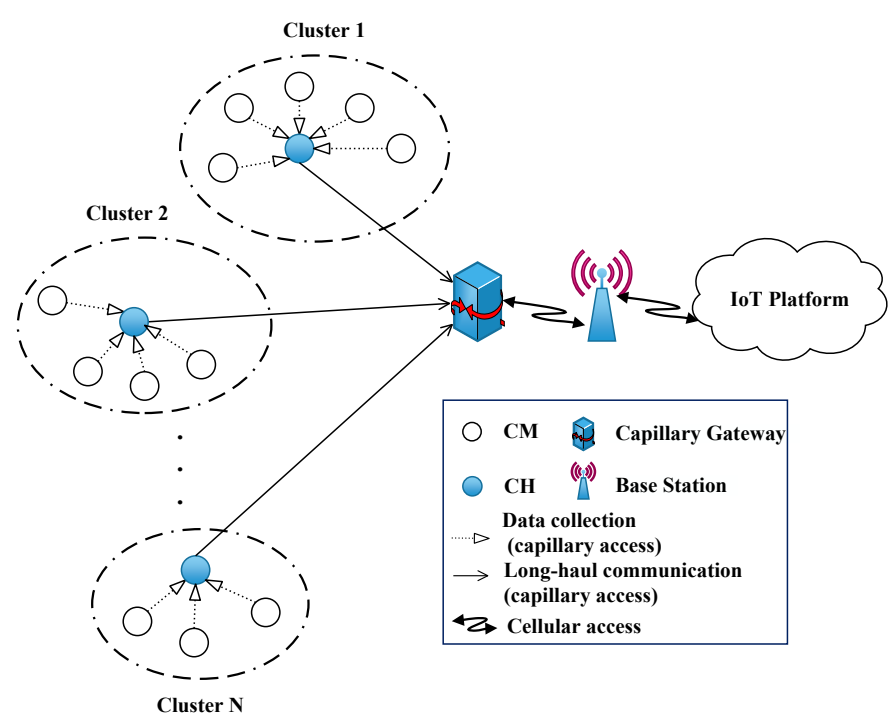

Fig. 1. System model

The system model shown in Fig. 1 considers a capillary networks for IoT systems with $\mathcal{N}_{\text {total }}$ power-constrained wireless devices: $\mathcal{N}_{C H} \mathrm{CHs}$ and $\mathcal{N}_{C M} \mathrm{CMs}$, where $\mathcal{N}_{\text {total }}=$ $\mathcal{N}_{C H}+\mathcal{N}_{C M}$. All devices are randomly distributed over the capillary networks with following assumptions:

- All wireless devices are homogeneous and energy constrained.

- All wireless devices are aware of their geographical locations and residual energies.

- All wireless devices are equipped with short-range local area wireless radio, e.g. IEEE 802.15.4.

- All devices are capable of operating in data collection and aggregation mode.

- A static capillary gateway is equipped with two radio interfaces: the local area capillary radio to communicate with the capillary networks and the cellular radio to communicate with the IoT systems.

The transmission is operated in two phases of one round as shown in Fig. 2: set-up phase and steady state phase. During the set-up phase, the gateway executes the clustering algorithm and informs every device with its role. The steady state phase consists of several time frames. During one time frame, all devices collect and transmit data in time division multiple access (TDMA) scheduling. Denote the duration of one time frame to be $t_{\Delta}$ and number of time frames of the steady state phase in one round to be $\mathcal{N}_{\text {frame }}$. In general, compared with the duration of the steady state phase, the duration of set-up phase is much shorter, which can be omitted, therefore the duration time of one round is $T_{\text {round }}=t_{\Delta} \times \mathcal{N}_{\text {frame }}$.

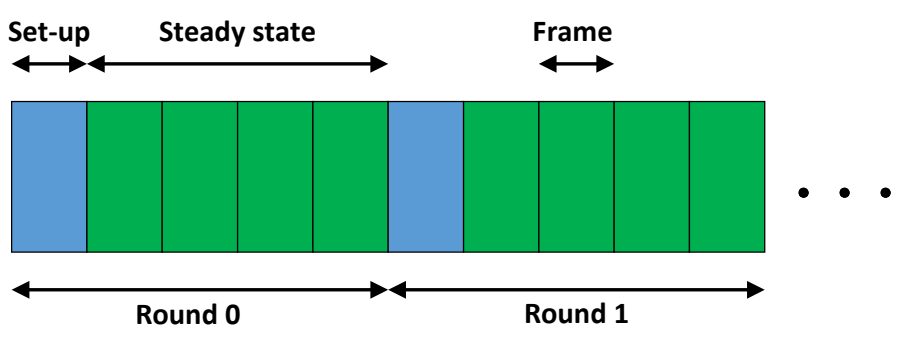

Fig. 2. Transmission structure in cluster-based IoT system

\section{B. Energy model}

Assume the connection between CMs and $\mathrm{CHs}$ as well as the connection between $\mathrm{CHs}$ and the gateway are all modelled by the flat Rayleigh-fading channel. And the modulation scheme is binary phase-shift keying (BPSK). Assume the data packet is $L$ bits for all devices. With these assumptions, when a source device transmit data to a destination device, the transmission energy consumption per bit $\bar{E}_{b}$ can be approximated as [12]

$$
\bar{E}_{b}=\frac{(4 \pi)^{2} M_{l} N_{f} N_{0} d^{\kappa}}{G_{T} G_{R} \lambda^{2} P_{b}}
$$

where $d$ is the transmission distance, $\lambda$ is the carrier wavelength, $\kappa$ is the path loss coefficient, $M_{l}$ is the link margin, $N_{f}$ is the receiver noise figure, $N_{0}$ is the single-sided thermal noise power spectral density, $G_{T}$ and $G_{R}$ are the transmitter and receiver antenna gain respectively, $P_{b}$ is the required bit error ratio (BER).

We adopt the energy consumption per bit of the source device in [12], which is expressed as below

$$
E_{b t}=(1+\alpha) \bar{E}_{b}+\frac{P_{c t}}{R_{b}},
$$

where $\alpha$ is a constant about the drain efficiency of radio frequency $(\mathrm{RF})$ power amplifier and the modulation, $P_{c t}$ is the power consumption of transmission circuit and $R_{b}$ is the transmission bit rate.

On the other hand, when a destination device receives data from a source device, the energy consumption is

$$
E_{b r}=\frac{P_{c r}}{R_{b}}
$$

where $P_{c r}$ is the power consumption of receiver circuit.

\section{Problem formulation}

In this work, we focus on the intra-cluster and inter-cluster energy consumption of data transmission in all rounds.

For every round, each $\mathrm{CM}$ transmits data to its $\mathrm{CH}$ based on TDMA scheme in each cluster, thus the overall intra-cluster energy consumption for the $i$-th cluster is,

$$
\begin{aligned}
& E_{\text {intra }}(i)=L \sum_{j=1}^{n_{i}} E_{b t}(j)+n_{i} L E_{b r}(i) \\
& =L \sum_{j=1}^{n_{i}}\left[(1+\alpha) \frac{(4 \pi)^{2} M_{l} N_{f} N_{0} d_{j}^{\kappa}}{G_{T} G_{R} \lambda^{2} P_{b}}+\frac{P_{c t}}{R_{b}}\right]+\frac{n_{i} L P_{c r}}{R_{b}},
\end{aligned}
$$


where $d_{j}$ is the distance between $\mathrm{CM} j$ and $\mathrm{CH} i$, and $n_{i}$ is the CMs number of the $i$-th $\mathrm{CH}$.

$\mathrm{CHs}$ are responsible for data aggregation. As referred to [13], assume the data aggregation scheme is uniform aggregation, and the aggregation factor is $\gamma_{a g g}$. Then the packet size after data aggregation of $\mathrm{CH} i$ in this phase is

$$
L_{a g g}(i)=\gamma_{a g g} n_{i} L
$$

The data aggregation energy consumption of $\mathrm{CH} i$ is

$$
E_{a g g}(i)=L_{a g g}(i) \bar{E}_{a g g},
$$

where $\bar{E}_{a g g}$ is the energy consumption per bit in data aggregation phase and depends on the algorithm complexity.

After data collection and aggregation, $\mathrm{CHs}$ transmit the aggregated data to the capillary gateway, therefore, the intercluster energy consumption of $\mathrm{CH} i$ is

$$
\begin{aligned}
& E_{\text {inter }}=\sum_{i=1}^{\mathcal{N}_{C H}} L_{a g g}(i)\left(E_{b t}(i)+E_{b r}\right) \\
& =\sum_{i=1}^{\mathcal{N}_{C H}} L_{a g g}(i)\left[(1+\alpha) \frac{(4 \pi)^{2} M_{l} N_{f} N_{0} d_{i}^{\prime \kappa}}{G_{T} G_{R} \lambda^{2} P_{b}}+\frac{P_{c t}+P_{c r}}{R_{b}}\right],
\end{aligned}
$$

where $d_{i}^{\prime}$ is the distance between $\mathrm{CH} i$ and the capillary gateway.

Therefore, the overall network energy consumption can be derived as

$$
E_{\text {net }}=E_{\text {inter }}+\sum_{i=1}^{\mathcal{N}_{C H}}\left(E_{\text {intra }}(i)+E_{\text {agg }}(i)\right) .
$$

Correspondingly, we can also obtain the energy consumption of $\mathrm{CM} j$ denoted by $E(j)$ in this round,

$$
E(j)=L\left[(1+\alpha) \frac{(4 \pi)^{2} M_{l} N_{f} N_{0} d_{j}^{\kappa}}{G_{T} G_{R} \lambda^{2} P_{b}}+\frac{P_{c t}}{R_{b}}\right] .
$$

Similarly, the energy consumption of $\mathrm{CH} i$ denoted by $E(i)$ in this round is,

$$
\begin{aligned}
E(i)= & L \frac{P_{c r}}{R_{b}}+L_{a g g} \bar{E}_{a g g} \\
& +L_{a g g}\left[(1+\alpha) \frac{(4 \pi)^{2} M_{l} N_{f} N_{0} d_{i}^{\prime \kappa}}{G_{T} G_{R} \lambda^{2} P_{b}}+\frac{P_{c t}}{R_{b}}\right] .
\end{aligned}
$$

In addition, the capillary network lifetime is defined as the duration from the deployment of the capillary network to the time that the battery of the first device is fully drained [14]. In this paper, we use average estimated average network lifetime as the fitness value of the proposed algorithm. Denote the residual energy of device $n$ to be $E_{r}(n)$. Then the estimated lifetime of device $n$ which denoted by $T_{n}$ is,

$$
T_{n}=\frac{E_{r}(n)}{E(n)} \text {. }
$$

The average estimated network lifetime $T_{n e t}^{r}$ in the round $r$ is

$$
T_{n e t}^{r}=\frac{\sum_{n=1}^{\mathcal{N}_{\text {total }}} T_{n}^{r}}{\mathcal{N}_{\text {total }}}
$$

The research problem is to find the optimal $\mathrm{CHs}$ coalition $\Lambda=\left\{C H_{1}^{r}, \cdots, C H_{\mathcal{N}_{C H}}^{r}\right\}$ at each round in order to optimize $T_{n e t}^{r}$, which is expressed as

$$
\underset{\Lambda}{\operatorname{maximize}} T_{n e t}^{r}
$$

\section{Description AND ANALYsis of QPSO Algorithm}

\section{A. Quantum Particle Swarm Optimization}

PSO is an evolutionary computing technique based on the bird flocking principle. In PSO, a swarm consists of several particles and each particle represents a candidate solution to the optimization problem.

QPSO uses quantum coding mechanism to encode each particle by a quantum bit. In [15], a quantum bit is defined as a pair of composite numbers $(\alpha, \beta)$, where $|\alpha|^{2}+|\beta|^{2}=1$ and $\alpha>0, \beta>0 .|\alpha|^{2}$ gives the probability that the quantum bit is found in ' 0 ' state and $|\beta|^{2}$ gives the probability that the quantum bit is found in ' 1 ' state. Then the quantum velocity of the particle $m$ at generation $t$ is defined as

$$
\mathbf{v}_{m}^{t}=\left[\begin{array}{cccc}
\alpha_{m 1}^{t} & \alpha_{m 2}^{t} & \cdots & \alpha_{m R}^{t} \\
\beta_{m 1}^{t} & \beta_{m 2}^{t} & \cdots & \beta_{m R}^{t}
\end{array}\right],
$$

where $m \in[1,2, \cdots, h], h$ is the number of particles and $R=\mathcal{N}_{\text {cand }}$ which indicates the number of $\mathrm{CH}$ candidates and will be further explained in Section III-B. Since $\beta_{m n}=$ $\sqrt{1-\alpha_{m n}^{2}}$, we can simplify Eq. (14) as

$$
\mathbf{v}_{m}^{t}=\left[\begin{array}{llll}
\alpha_{m 1}^{t} & \alpha_{m 2}^{t} & \cdots & \alpha_{m R}^{t}
\end{array}\right] .
$$

The quantum particle position according to Eq. (15) can be expressed as

$$
x_{m n}^{t}=\left\{\begin{array}{lll}
1 & \text { if } \quad & \delta_{m n}>\left(v_{m n}^{t}\right)^{2} \\
0 & \text { if } \quad & \delta_{m n} \leq\left(v_{m n}^{t}\right)^{2}
\end{array},\right.
$$

where $\delta_{m n} \in[0,1]$ is a uniform random number. In this paper, the quantum position indicates whether the device $n$ is a $\mathrm{CH}$ in particle $m: x_{m n}^{t}=1$ represents that device $n$ in particle $m$ is a $\mathrm{CH}$ at generation $t$; otherwise, device $n$ in particle $m$ is a $\mathrm{CM}$ at generation $t$. Therefore each particle in this paper represents a candidate solution of a particular $\mathrm{CHs}$ coalition, and the fitness value of each particle can then be obtained by Eq. (12).

Denote the fitness value of particle $m$ at generation $t$ to be $f_{m}^{t}$, then the local individual optimum fitness value (the best fitness value of particle $m) f_{m}^{p b e s t}$ and the corresponding local individual optimum position $\mathbf{p}_{m}$ is defined as below,

$$
\begin{gathered}
f_{m}^{p b e s t}=\max \left\{f_{m}^{1}, f_{m}^{2}, \cdots, f_{m}^{t}\right\} \\
\mathbf{p}_{m}=\mathbf{x}_{m}^{\text {pbest }}
\end{gathered}
$$


Similarly, the global optimum fitness value (the best fitness value of all particles) $f_{\text {gbest }}$ and the corresponding global optimum position $\mathbf{p}_{g}$ is defined as below,

$$
\begin{gathered}
f_{\text {gbest }}=\max \left\{f_{1}^{\text {pbest }}, \cdots, f_{m}^{\text {pbest }}, \cdots, f_{h}^{\text {pbest }}\right\} \\
\mathbf{p}_{g}=\mathbf{p}_{\text {gbest }} .
\end{gathered}
$$

At generation $t+1$, the quantum rotation angle $\theta_{m n}^{t+1}$ is updated by

$$
\theta_{m n}^{t+1}=k_{1}\left(p_{m n}-x_{m n}^{t}\right)+k_{2}\left(p_{g n}-x_{m n}^{t}\right),
$$

where $k_{1}$ and $k_{2}$ are two positive learning factors of cognitive and social acceleration factors, respectively. As referred to [16], we set $k_{1}=\frac{1}{5} \zeta_{1}$ and $k_{2}=\frac{4}{5} \zeta_{2}$ where $\zeta_{1}$ and $\zeta_{2}$ are Gaussian distributed random numbers with zero mean and unit variance.

If $\theta_{m n}^{t+1} \neq 0$, the updated velocity of quantum particle $m$ at $t+1$ generation is,

$$
v_{m n}^{t+1}=\left|v_{m n}^{t} \times \cos \theta_{m n}^{t+1}-\sqrt{1-\left(v_{m n}^{t}\right)^{2}} \times \sin \theta_{m n}^{t+1}\right| .
$$

If $\theta_{m n}^{t+1}=0$ and $r<c_{1}$, the updated velocity of quantum particle $m$ at $t+1$ generation is,

$$
v_{m n}^{t+1}=\sqrt{1-\left(v_{m n}^{t}\right)^{2}},
$$

where $r$ is a uniform random number between 0 and 1 , and $c_{1}$ is a constant which refers to the mutation probability, $c_{1} \in$ $[0,1 / R]$.

\section{B. QPSO based CHs selection clustering algorithm}

The CHs selection process based on QPSO is executed by the capillary gateway and is summarized in Algorithm 2. The clustering procedure is summarized in Algorithm 1. Note that in order to ensure that the selected $\mathrm{CHs}$ are of sufficient energy, $\mathrm{CH}$ candidates are selected beforehand. Denote the $\mathrm{CH}$ candidates percentage to be $\mathcal{P}_{C H}$, then the number of $\mathrm{CH}$ candidates is $\mathcal{N}_{\text {cand }}=\mathcal{P}_{C H} \times \mathcal{N}_{\text {total }}$. Only first $\mathcal{N}_{\text {cand }}$ devices with highest residual energy level among the scenario are eligible to be $\mathrm{CHs}$ for this round. If the residual energy of different nodes are the same, the long-haul distance is considered, i.e. devices close to the gateway is more likely to be selected as $\mathrm{CH}$ candidates.

\section{Simulation Results}

Assume 200 devices powered by AAA Carbon-zinc battery $(1.1 \mathrm{~V}, 320 \mathrm{mAh})$ [17] are randomly distributed located within a square with $500 \mathrm{~m}$ side length. The gateway is $250 \mathrm{~m}$ away from the closest device. The system parameters are given in Table I.

In terms of battery energy conversion, We use following battery capacity model [18] to convert the energy consumption $E(J)$ to Watt-hours $E(W h)$,

$$
\begin{aligned}
& E(J)=Q(m A h) \times V(v) \times 3600 \\
& E(W h)=Q(m A h) \times V(v) \\
& E(W h)=\frac{E(J)}{3600} .
\end{aligned}
$$

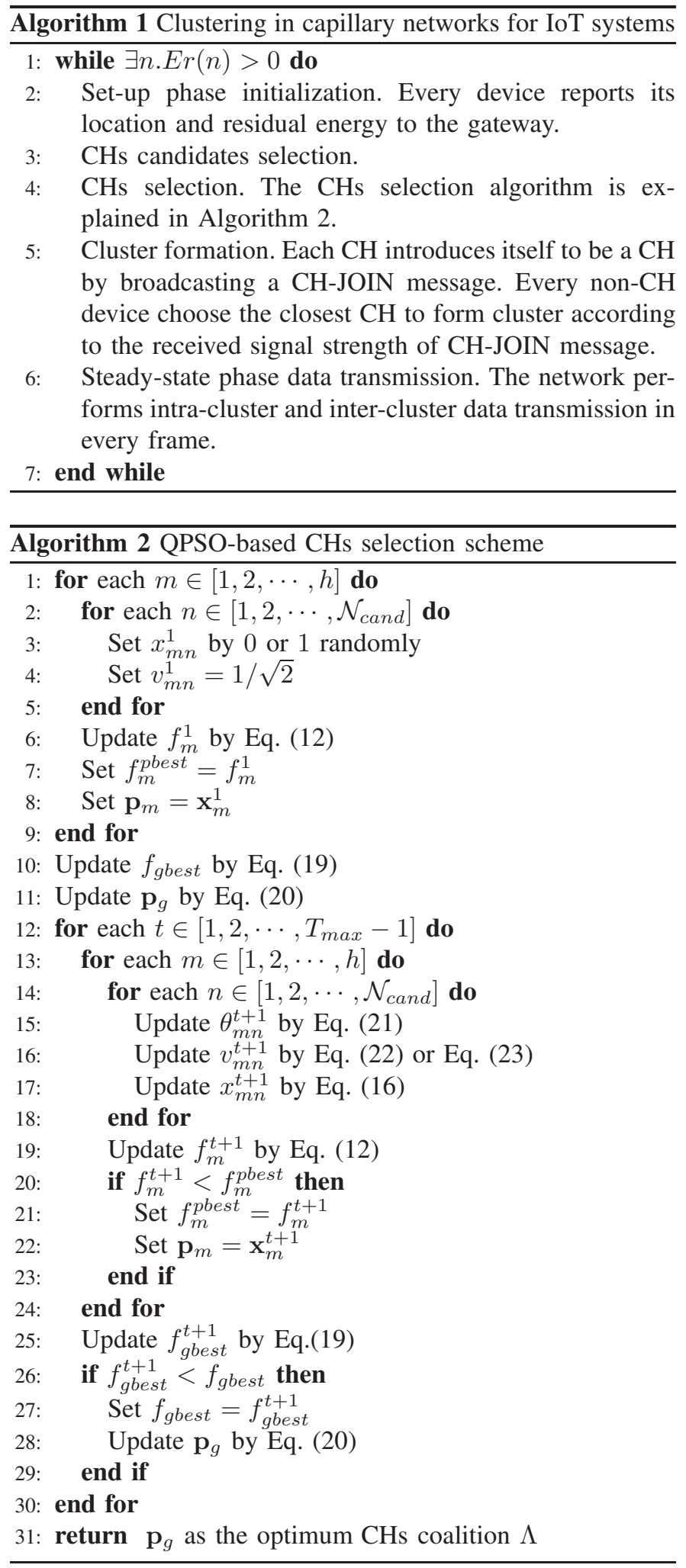

By Eq. (24), the initial battery capacity of all devices is $1268 \mathrm{~J}$ in energy and $0.35 \mathrm{Wh}$ in Watt-hours in the defined scenario.

In this work, we compare the proposed QPSO algorithm with three evolutionary algorithms: PSO [6], BPSO [7] and QGA [8]. 
TABLE I

SYSTEM PARAMETERS

\begin{tabular}{|c|c|c|}
\hline$M_{l}=40 d B$ & $N_{f}=10 d B$ & $N_{0}=-171 \mathrm{dBm} / \mathrm{Hz}$ \\
\hline$\kappa=2.5$ & $G_{T} G_{R}=5 d B i$ & $\lambda=0.12 \mathrm{~m}$ \\
\hline$P_{b}=10^{-3}$ & $\alpha=0.47$ & $R_{b}=10 \mathrm{kbps}$ \\
\hline$P_{c t}=98.2 \mathrm{~mW}$ & $P_{c r}=112.5 \mathrm{~mW}$ & $L=100 \mathrm{bit}$ \\
\hline$\gamma_{a g g}=0.5$ & $\mathcal{N}_{\text {frame }}=24$ & $\mathcal{P}_{C H}=0.2$ \\
\hline$t_{\Delta}=30 \mathrm{~min}$ & $E_{a g g}==5 \mathrm{~nJ} / \mathrm{bit}$ & \\
\hline
\end{tabular}

First, one of the main difficulties of applying an evolutionary algorithm to a given problem is to decide an appropriate set of parameter values [19]. In order to find the optimum particle number and generation for the assumed scenario, we compare in Fig. 3 the fitness value of different number of particles for the proposed QPSO algorithm. Obviously, the fitness value increases dramatically in the first 50 generations as all particles are flying in the solution space moving towards the optimum global fitness value. Then the fitness value converges to the similar points because all particles have found global optimum solution. Note that the fitness values of QPSO algorithm with a different particle number remains constant after 200 generations. Most importantly, with the increase of particles number from 30 to 100 , the improvement of fitness value is not significant. In addition, more particles means more timeconsuming computation in evolutionary algorithms. Therefore, we set the optimum particles number to be 30 and generation to be 200 for all the algorithms simulated in this paper.

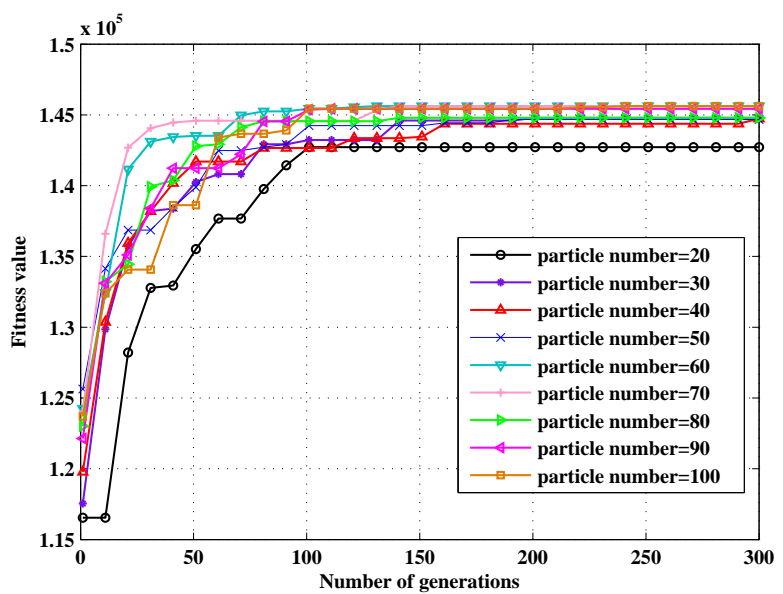

Fig. 3. Number of generations vs. fitness value

Fig. 4 depicts the fitness value of the four algorithms against the number of generations. Similar to Fig. 3, all algorithms can converge to the optimum fitness value with the increase of generation. It is obvious that the proposed QPSO algorithm can find the best cluster coalitions compared with other three algorithms by the best fitness value in Eq. (12).

Moreover, in Fig. 5 we compare the overall residual battery capacity of all devices among the scenario. It can be seen that the proposed QPSO algorithm can prolong the network lifetime compared with PSO, BPSO and QGA. In particular, the overall residual battery capacity decreases significantly

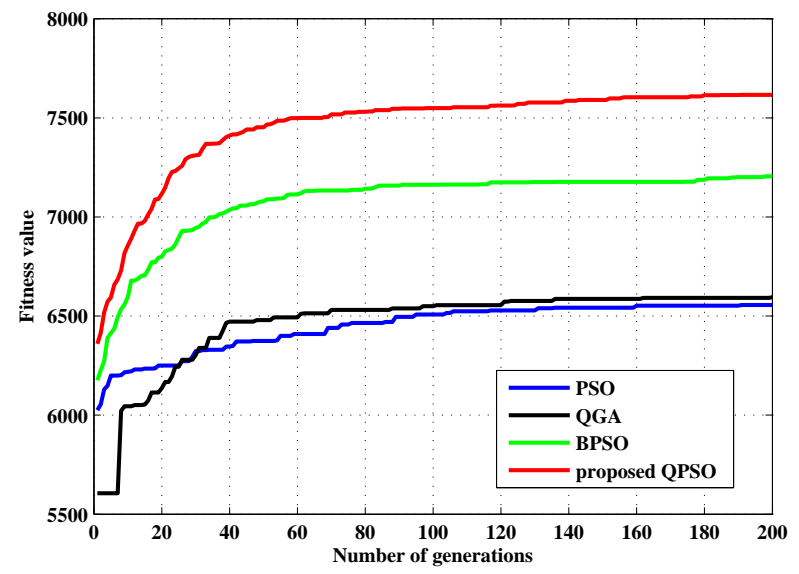

Fig. 4. Number of generations vs. fitness value

after 2000 hours, but QPSO outperforms other algorithms due to optimum $\mathrm{CHs}$ selection and more evenly distributed energy consumption.

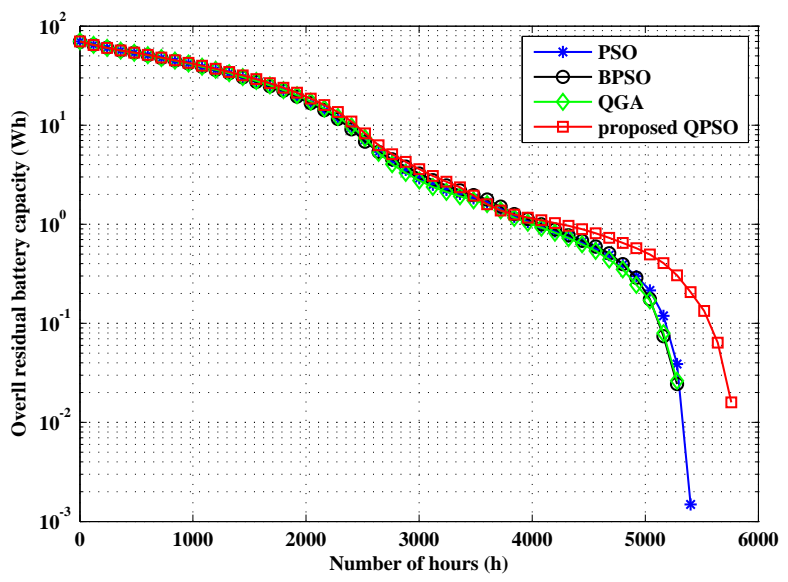

Fig. 5. Number of hours vs. overall residual battery capacity

Fig. 6 shows the number of alive nodes of the four algorithms with respect to the number of hours. The first device died after about 2000 hours for all algorithms, followed by an increasing number of dead nodes from 2000 hours to 4500 hours. This is because the devices close to the gateway is more likely to be selected as $\mathrm{CHs}$ and run out of energy quicker, and the long-haul transmission requires more energy consumption after the death of those devices. In addition, almost all devices of PSO, BPSO and QGA enter dead status at 5500 hours, however, the proposed QPSO algorithm can prolong the network lifetime of the assumed scenario by another 500 hours (about 10\%).

Finally, we showed that the number of $\mathrm{CHs}$ of all algorithms in Fig. 7. Similar to Fig. 6, the number of $\mathrm{CHs}$ decreases with less alive nodes after 2000 hours. Although the QPSO outperforms other algorithms in Fig. 4 - Fig. 6, the number of 


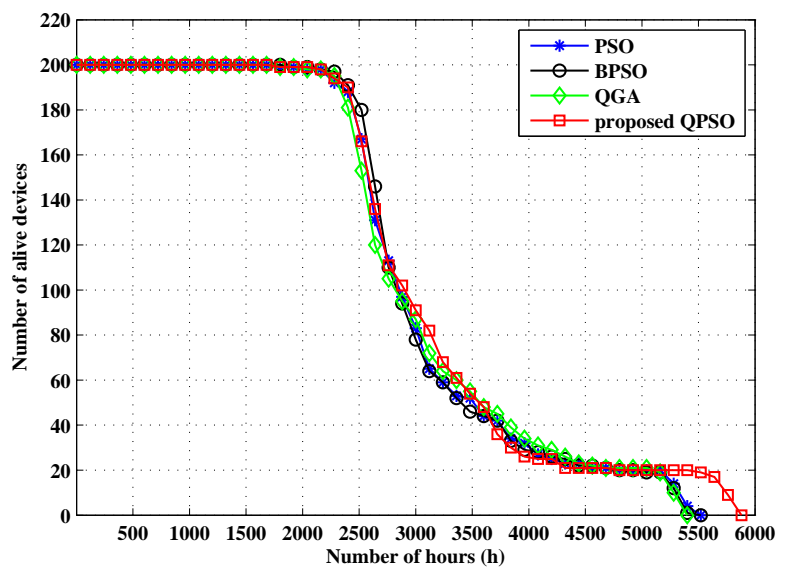

Fig. 6. Number of hours vs. number of alive nodes

CHs selected by the proposed QPSO QPSO algorithm is not the maximum at most time. This result indicates that higher number of $\mathrm{CHs}$ is not necessary.

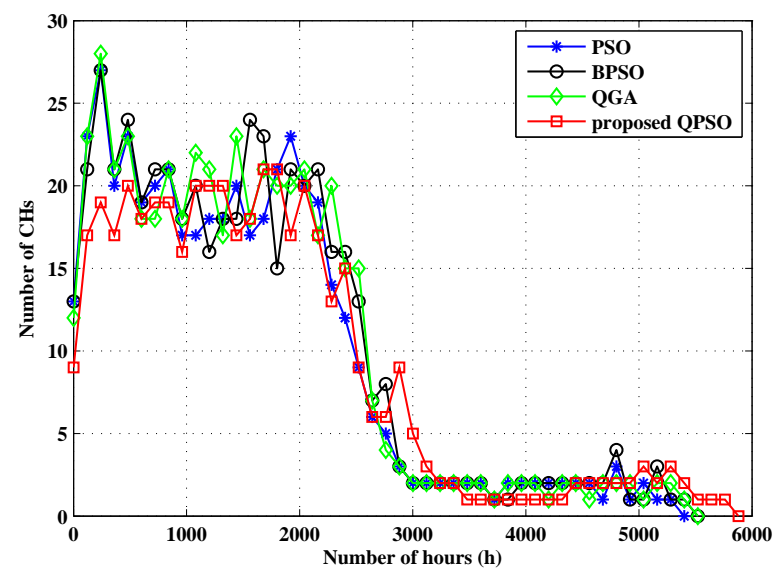

Fig. 7. Number of hours vs. number of $\mathrm{CHs}$

\section{CONCLUSIONS}

In this paper, we investigate cluster structure design using QPSO with the aim of network lifetime longevity in clusterbased IoT systems. We show that the $\mathrm{CHs}$ selection plays an important role in data forwarding. The QPSO algorithm is proposed in order to select the optimum $\mathrm{CHs}$ coalition. Simulation results show that the proposed QPSO scheme outperforms PSO, BPSO and QGA algorithms in terms of network lifetime by approximately $10 \%$.

\section{REFERENCES}

[1] D. Liu, L. Wang, Y. Chen, M. Elkashlan, K. Wong, R. Schober, and L. Hanzo, "User association in $5 \mathrm{~g}$ networks: A survey and an outlook," CoRR, vol. abs/1509.00338, 2015. [Online]. Available: http://arxiv.org/abs/1509.00338

[2] Y. Li, K. Chai, Y. Chen, and J. Loo, "Duty cycle control with joint optimisation of delay and energy efficiency for capillary machineto-machine networks in $5 \mathrm{~g}$ communication system," Transactions on Emerging Telecommunications Technologies, vol. 26, pp. 56-69, 2015.

[3] V. Misic, J. Misic, and D. Nerandzic, "Extending lte to support machinetype communications," in Communications (ICC), 2012 IEEE International Conference on, 2012, pp. 6977-6981.

[4] E. Falkenauer, Genetic Algorithms and Grouping Problems. New York, NY, USA: John Wiley \& Sons, Inc., 1998.

[5] F. Lin, C. Kao, and C. Hsu, "Applying the genetic approach to simulated annealing in solving some np-hard problems," Systems, Man and Cybernetics, IEEE Transactions on, vol. 23, no. 6, pp. 1752-1767, Nov 1993.

[6] N. Latiff, C. Tsimenidis, and B. Sharif, "Energy-aware clustering for wireless sensor networks using particle swarm optimization," in Personal, Indoor and Mobile Radio Communications, 2007. PIMRC 2007. IEEE 18th International Symposium on, 2007, pp. 1-5.

[7] N. Latiff, C. Tsimenidis, B. Sharif, and C. Ladha, "Dynamic clustering using binary multi-objective particle swarm optimization for wireless sensor networks," in Personal, Indoor and Mobile Radio Communications, 2008. PIMRC 2008. IEEE 19th International Symposium on, Sept 2008, pp. 1-5.

[8] J. Guo, L. Sun, X. Du, and L. Liu, "Clustering protocol based on data aggregating scheme for wireless sensor networks," WIT transaction on information and communication technology, vol. 51, pp. 379-386, 2014.

9] W. Heinzelman, A. Chandrakasan, and H. Balakrishnan, "Energyefficient communication protocol for wireless microsensor networks,' in System Sciences, 2000. Proceedings of the 33rd Annual Hawaii International Conference on, 2000, pp. 10 pp. vol.2-.

[10] J. Cao, T. Zhang, Z. Zeng, Y. Chen, and K. Chai, "Multi-relay selection schemes based on evolutionary algorithm in cooperative relay networks,' International Journal of Communication Systems, 2014.

[11] M. Tarhani, Y. Kavian, and S. Siavoshi, "Seech: Scalable energy efficient clustering hierarchy protocol in wireless sensor networks," Sensors Journal, IEEE, vol. 14, no. 11, pp. 3944-3954, Nov 2014.

[12] S. Cui, A. Goldsmith, and A. Bahai, "Energy-efficiency of mimo and cooperative mimo techniques in sensor networks," Selected Areas in Communications, IEEE Journal on, vol. 22, no. 6, pp. 1089-1098, 2004.

[13] Z. Huang, H. Okada, K. Kobayashi, and M. Katayama, "A study on cluster lifetime in multi-hop wireless sensor networks with cooperative miso scheme," Communications and Networks, Journal of, vol. 14, no. 4, pp. 443-450, Aug 2012.

[14] T. Himsoon, W. Siriwongpairat, Z. Han, and K. Liu, "Lifetime maximization by cooperative sensor and relay deployment in wireless sensor networks," in Wireless Communications and Networking Conference, 2006. WCNC 2006. IEEE, vol. 1, 2006, pp. 439-444.

[15] H. Gao, J. Cao, and M. Diao, "A simple quantum-inspired particle swarm optimization and its application," Information Technology Journal, vol. 10, 2011

[16] J. Cao, T. Zhang, Z. Zeng, and D. Liu, "Interference-aware multi-user relay selection scheme in cooperative relay networks," in Globecom Workshops (GC Wkshps), 2013 IEEE, Dec 2013, pp. 368-373.

[17] A. A. Battery, "Find the energy contained in standard battery sizes," http://www.allaboutbatteries.com/Energy-tables.html, 2011.

[18] RapidTables, "How to convert mah to wh," http://www.rapidtables.com/ convert/electric/mah-to-wh.htm, 2014.

[19] F. Lobo, C. Lima, and Z. Michalewicz, Parameter Setting in Evolutionary Algorithms, 1st ed. Springer Publishing Company, Incorporated, 2007. 